\title{
Saudi Aramco's Real-Time Tar Mat and Oil Reservoir Characterization via GC-ROX: Application Examples
}

\author{
Peter J. Jones, Henry I. Halpern and Salman M. Al-Qathami, Saudi Aramco.
}

Saudi Aramco has developed fully commercial methods to quantitatively assess reservoir oil quality, productivity, water saturation, and tar identification and quantification from residual hydrocarbon staining on drill cuttings. The patented methods are known collectively as Pyrolytic Oil-Productivity Index (POPI) Technology. Unlike results from wellsite logging tools, which provide an indirect assessment of the rock properties, pore system, and fluid properties in a reservoir, pyrolytic methods provide a direct assessment of the residual hydrocarbons present in rock samples and can be used to assess connectivity with the active fluid system in the reservoir.

POPI instrumentation and methods can accurately quantify tar volumes over a wide range of concentrations, and are routinely applied to drilled cuttings in real-time at the wellsite to assist in geosteering horizontal development wells. POPI data are integrated with other well data to confirm correct well placement early in the drilling process. This provides better success in meeting well design objectives while reducing lost rig time for unnecessary testing, or the drilling of additional side-track wells.

Saudi Aramco has exploited this technology in the Kingdom through the commercialization of services, which to date, have been provided in real-time at over 140 horizontal wells and over 375 wells including laboratory studies. A key step in commercializing Saudi Aramco's POPI Technology was achieved with the development of an advanced geochemical analysis and modeling application known as GC-ROX - Geochemical Residual Oil eXpert. GC-ROX is a first-of-a-kind software that integrates data validation, organization, calibration, modeling, calculation, visualization, interpretation, integration with other data sources, and results delivery. The software accomplishes the delivery of high-level geochemical interpretations in "real-time", at the well site, by field personnel and can interface directly with real-time data servers, exchanging data via the industry standard WITSML protocol.

GC-ROX can be applied to optimize oil field development in a variety of ways. One of the most common issues that it has been used to address is the identification and quantification of tar mats. The results are used in combination with results from NMR and formation pressure while drilling tools (FPWD) while geosteering to assess fluid moveability to ensure that well performance objectives are met. The software can also facilitate the assessment of oil reservoir productivity to assist in maximizing reservoir contact in the most productive portion of the reservoir. A key capability of GC-ROX is the ability to assess drilling mud contamination effects present in samples and correct the output to provide improved interpretations of reservoir quality. 